\section{KOMPASS}

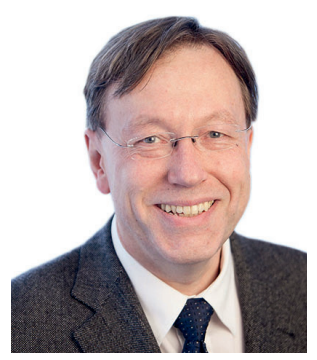

Helmut Frohnhofen

Universität Witten/Herdecke, Fakultät für Gesundheit, Lehrstuhl für Geriatrie, Witten, Deutschland

In dieser aktuellen Ausgabe des KARGER KOMPASS PNEUMOLOGIE werden wieder wichtige, alltagsrelevante Themen aus der Pneumologie unter dem Themenfokus «Lungenalter messen und berücksichtigen» vorgestellt. So beschäftigt sich die Übersichtsarbeit von Shikma Katz und Kollegen mit der dem Einfluss der Körperposition auf das Ergebnis einer Lungenfunktionsprüfung. Dieses Thema hat insofern Alltagsrelevanz, als nicht jeder Patient mit Lungenproblemen eine sitzende Position einnehmen kann. Die Metaanalyse zeigt klar, dass eine sitzende Körperposition bei der Lungenfunktionsprüfung anzustreben ist, dass aber Patienten mit Rückenmarksverletzung in liegender Position bessere Werte erbringen.

Die Ergebnisse einer Lungenfunktionsprüfung haben gerade nach thorakalen Eingriffen prognostische Relevanz. In seinem Wissenstransfer zeigt Khosro Hekmat, dass die Einsekundenkapazität und die Diffusionskapazität Prädiktoren für relevante Endpunkte sind.

Amiodaron wird als Antiarrhythmikum mit etwa 5\% häufig verordnet. Amiodaron kann gerade im multimorbiden Kontext älterer Menschen auch seltene Erkrankungen der Lunge wie eine Lipoidpneumonie hervorrufen. Eine solche Kasuistik einschließlich des Verlaufes und der Effekte der Behandlung werden im Fallbeispiel dieser Ausgabe von llektra Voulgareli et al. diskutiert.

Interessant ist das Ergebnis einer vergleichenden Untersuchung an Neonaten mit

\title{
Lungenalter messen und berücksichtigen
}

Atemnotsyndrom, die David Brandt kommentiert hat. Aufgrund der hohen Übereinstimmungsrate zwischen röntgenologischer Bildgebung und einer Ultraschalluntersuchung der Lunge könnte das letztere Verfahren als weniger belastend und schneller durchführbar zu einer Veränderung etablierter Standards führen.

Die Abklärung von Hämoptysen erfolgt üblicherweise mittels endoskopischer Diagnostik. Der optimale Zeitpunkt für die Durchführung einer Bronchoskopie ist aber unklar. Die von Manfred Wagner bewertete Studie zeigt, dass bei frühzeitigem Durchführen dieser Untersuchung bei moderater Blutungsintensität häufiger der Ort der Blutung lokalisiert werden kann, jedoch hat eine frühzeitig durchgeführte Bronchoskopie bezüglich der ätiologischen Klärung keine Vorteile zu bieten. Daher relativiert sich die Dringlichkeit einer bronchoskopischen Diagnostik bei Hämoptysen. Maligne Pleuraergüsse sind ein häufiges Problem in der Thoraxonkologie. Stefanie Keymel geht in ihrem Wissenstransfer darauf ein, dass die Pleurakatheteranlage mit zusätzlicher Talkuminstillation zur Pleurodese effektiver zu sein scheint, als die alleinige Anlage eine Pleuradrainage mittels Pleurakatheter. Die Erfolgsraten waren zwar mit 43\% bzw. 23\% für beide Verfahren moderat, jedoch war die Lebensqualität der mit einer Talkumpleurodese behandelten Patienten besser.

Dyspnoe ist ein häufiges Symptom pulmonaler und kardialer Erkrankungen. Ein weiterer Kommentar zeigt die hohe Koinzidenz von Koronarerkrankung und COPD. Daher sollten Koronarpatienten unter dem Aspekt einer COPD und COPD-Patienten unter dem Aspekt einer Koronarerkrankung untersucht werden.

Das Atemnotsyndrom des Erwachsenen ist ein aktuelles Problem in der Intensivmedizin. Die additive Gabe von künstlichem Surfactant hat jedoch eher enttäuscht. Es fand sich kein Einfluss auf die Oxygenierung und die Mortalität Betroffener, so dass die Surfctantgabe auch angesichts ihrer Kosten kritisch gesehen werden muss. Näheres dazu lesen Sie im Artikel von Georgios Sofianos.

Wie unterschiedlich die Entität Bronchialkarzinom auf genetischer Ebene ist, zeigt eine weitere Studie, der sich Susanne Lang in ihrem Beitrag widmet. Dabei fanden sich zwischen verschiedenen Subtypen des Bronchialkarzinoms auf genetischer Ebene nur wenige Gemeinsamkeiten. Daraus ergibt sich die Frage nach der Notwendigkeit einer gezielteren Ausrichtung von Therapieregimen. Hier sind weitere Forschungen erforderlich. Dieses Heft bietet einen interessanten Einblick in spezielle, aber relevante Themen der täglichen pneumologischen Praxis und hilft hierdurch bei der manchmal nicht einfachen Entscheidungsfindung.

Eine anregende Lektüre wünscht Ihnen

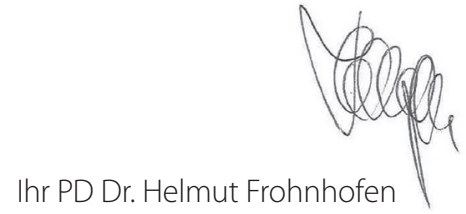

\section{KARGER}

Fax +497614520714

information@karger.com

www.karger.com
๑๑ 2019 S. Karger GmbH, Freiburg

Accessible online at: www.karger.com/kkp
PD Dr. Helmut Frohnhofen

Universität Witten/Herdecke

Fakultät für Gesundheit, Lehrstuhl für Geriatrie

Alfred-Herrhausen-Straße 50, 58448 Witten, Deutschland

helmut.frohnhofen@uni-wh.de 